\title{
Intrapleural perfusion thermo-chemotherapy for pleural effusion caused by lung carcinoma under VATS
}

\author{
Runlei $\mathrm{Hu}^{1}$, Hong Jiang ${ }^{1}$, Hu Li ${ }^{1}$, Dongshan Wei ${ }^{1}$, Guoqing Wang ${ }^{1}$, Shenglin $\mathrm{Ma}^{2}$ \\ ${ }^{1}$ Department of Thoracic Surgery, ${ }^{2}$ Department of Radiation Oncology, Hangzhou First People's Hospital, Nanjing Medical University, Hangzhou \\ 310006, China \\ Contributions: (I) Conception and design: R Hu, S Ma; (II) Administrative support: S Ma, H Jiang; (III) Provision of study materials or patients: \\ All authors; (IV) Collection and assembly of data: R Hu, D Wei, G Wang, H Li; (V) Data analysis and interpretation: R Hu, G Wang, H Li; (VI) \\ Manuscript writing: All authors, (VII) Final approval of manuscript: All authors. \\ Correspondence to: Dr. Shenglin Ma. Department of Radiation Oncology, Hangzhou First People's Hospital, Nanjing Medical University. Hangzhou \\ 310006, China. Email: mashenglin@medmail.com.cn.
}

Background: The aim of this study was to assess the effectiveness of intrapleural perfusion thermochemotherapy (IPTC) under video-assisted thoracoscopic surgery (VATS) for malignant pleural effusion (MPE) caused by lung carcinoma.

Methods: In this retrospective study, fifty-four patients with moderate or large amounts of ipsilateral MPE secondary to non-small cell lung cancer (NSCLC) underwent pleural biopsy and IPTC under VATS. IPTC was performed by perfusing the pleural cavity with $43.0{ }^{\circ} \mathrm{C}$ saline solution containing cisplatin $\left(200 \mathrm{mg} / \mathrm{m}^{2}\right)$ using a devised circuit through mechanical circulation for 60 minutes. Blood pressure, heart rate, oxygen saturation $\left(\mathrm{SpO}_{2}\right)$, and esophageal and rectal temperatures were monitored throughout the surgery. At the end of the perfusion, pleural biopsy was performed again for histological analysis.

Results: The temperature at the pleural surface was stabilized at $43{ }^{\circ} \mathrm{C}$, and pleural effusion was controlled in all patients. KPS scores increased in $89.3 \%$ of patients. No patient developed bone marrow suppression reactions with noticeable bleeding after treatment, and no liver and kidney malfunctions were observed. Apoptosis was detected by light and electron microscopy after IPTC. CEA markedly decreased in all patients 1 month after IPTC. The median survival time was 21.7 months, with a one-year survival rate of $74.1 \%$.

Conclusions: IPTC under VATS is a new, safe, less invasive and more effective approach for MPE caused by lung carcinoma.

Keywords: Video-assisted thoracoscopic surgery (VATS); lung carcinoma; pleural effusion; thermo-chemotherapy

Submitted Feb 13, 2017. Accepted for publication Apr 11, 2017.

doi: $10.21037 /$ jtd.2017.04.65

View this article at: http://dx.doi.org/10.21037/jtd.2017.04.65

\section{Introduction}

Malignant pleural effusion (MPE), defined by the presence of malignant cells in the pleural space, reflects advanced lung cancer and is associated with significant morbidity (1). MPE is considered a crucial negative factor in reducing the patient's quality of life (QOL) and survival (2). Many attempts to treat this disease, including surgery, have not been successful (3). Therefore, surgical therapy alone is generally not recommended. To improve prognosis, more extensive surgical procedures, such as pleuropneumonectomy and adjuvant chemotherapy, have been assessed, but no 'gold standard' for therapy has been established. Recently, the efficacy of pleural perfusion thermo-chemotherapy for MPE was reported $(4,5)$; it consists of co-administration of cytotoxic drugs (such as cisplatin) and thermotherapy with thoracotomy (6). However, this method may not be suitable for patients with poor pulmonary function and cardiac complications. Indeed, the rate of complications in patients with limited 
pulmonary function is significantly higher in patients undergoing thoracoscopy $(7,8)$. Therefore, in this study, we adopted IPTC for the video-assisted thoracoscopic surgery (VATS) approach in a minimally invasive manner for the treatment of pleural effusion caused by lung carcinoma.

\section{Methods}

From January 2004 to April 2013, fifty-four patients with MPEs caused by lung carcinoma but without distant metastasis underwent IPTC under VATS in our department. They were 45 to 75 years old, with a mean age of 64.9 years. All patients had been diagnosed with primary lung adenocarcinoma. The clinical stage of all patients was IV, since they had pleural metastasis (M1a) with no distant metastasis at the time of perfusion treatment. The patients underwent surgical pleural biopsy and received sequential perfusion treatment. This study was approved by the ethics committee of Hangzhou First People's Hospital (No. 101-01 Ethics).

\section{Therapy}

Intrapleural perfusion thermo-chemotherapy (IPTC) under VATS was performed generally according to the conventional method without thoracotomy (9). The patients underwent general anesthesia, and were intubated by suitable double lumen endotracheal tubes; using one-lung ventilation, each patient was placed in the lateral decubitus position. First, a 10 -mm port (port 1) was placed in the seventh intercostal space at the mid-axillary line. A second port was placed in the forth intercostal space at the anterior axillary line. Another port (port 3) was placed in the sixth intercostal space at the posterior axillary line. A thoracoscope was inserted through the first port, while the other two ports were utilized to perform biopsy and remove the fibrinous adhesions, thereby obtaining pleural biopsy tissues. Next, irrigation inlet (10 $\mathrm{mm})$ and outlet $(10 \mathrm{~mm})$ tubes were inserted through ports 1 and 2, and connected to a standard extra-corporal circuit. The circuits were primed with $3,000 \mathrm{~mL}$ of saline solution; the pleural space perfusion flow rate was maintained between 800 and $1,000 \mathrm{~mL} / \mathrm{min}$. A temperature probe was inserted and placed in the pleural cavity via port 3 . After confirming an intrapleural temperature of about $43^{\circ} \mathrm{C}$, cisplatin was administered at a total dose of $200 \mathrm{mg} / \mathrm{m}^{2}$. $500 \mathrm{mg}$ methylprednisolone and $8 \mathrm{mg}$ ondansetron were administered intravenously during IPTC to prevent pulmonary edema and vomiting, which are usually caused by thermotherapy. Once perfusion was completed, the solution in the thoracic cavity was completely removed, and a drainage tube placed via port 1 . Blood pressure, heart rate, $\mathrm{SpO}_{2}$, and esophageal and rectal temperatures were monitored throughout the surgery. At the end of the perfusion, pleural biopsy was performed for histological analysis. Serum carcinoembryonic antigen (CEA) levels before and after IPTC were determined.

\section{Histological analysis}

Tumor tissues were collected from pleural effusions and nodules. One part was fixed in $10 \%$ formalin and paraffin embedded, and stained with $\mathrm{H} \& \mathrm{E}$. The remaining part was fixed in $4 \%$ glutaraldehyde and cut into ultrathin sections for TEM.

\section{Follow-up visit}

Follow-up was performed every 3 months after hospital discharge.

\section{Results}

The temperature at the pleural surface was stabilized at $43^{\circ} \mathrm{C}$. The maximum temperature in the esophagus and rectum was less than $38.3^{\circ} \mathrm{C}$, despite the rise in pleural surface temperature.

\section{Clinical outcomes and toxicities after IPTC under VATS}

Efficacy was evaluated according to conventional criteria (10): (I) complete recovery (CR) was reflected by effusion clearance lasting for more than 4 weeks; (II) partial recovery (PR) indicated effusions reduced to less than $50 \%$, lasting for more than 4 weeks; CR and PR represented effective treatment. After IPTC, 54 cases showed CR, and 2 had PR; pleural effusion was controlled in $100 \%$ of patients. Symptoms, including chest distress, shortness of breath, cough, and chest pain, were noticeably ameliorated. KPS scores were increased in 48 cases (88.9\%) (Figure 1).

Changes in blood pressure and pulmonary artery pressure were mild. Two patients developed lung edema perioperatively, which was improved by induced diuresis, oxygen inspiration and steroid administration; 3 patients developed cardiac malfunction, which was ameliorated by heart stimulation and diuresis induction. No patient developed bone marrow suppression reaction with noticeable bleeding after treatment; no liver and kidney 

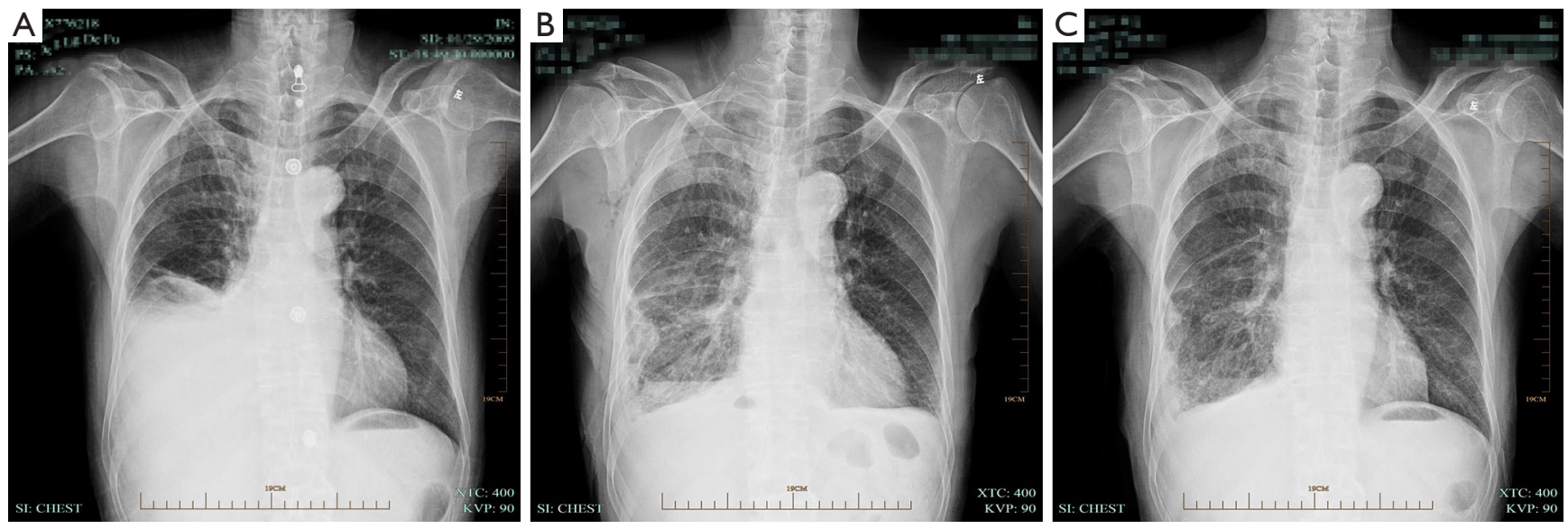

Figure 1 Chest X ray showing pleural effusion under control in 100\% of patients. (A) Before IPTC; (B) two months after IPTC; (C) six months after IPTC. IPTC, intrapleural perfusion thermo-chemotherapy.

Table 1 Clinical outcomes and toxicities before and after IPTC

\begin{tabular}{lcc}
\hline Variable & Before IPTC & After IPTC \\
\hline Arterial $\mathrm{O}_{2}$ tension $(\mathrm{mmHg})$ & $68.3 \pm 10.1$ & $72.4 \pm 9.5$ \\
Chemistry profile & & \\
White blood cells $\left(\times 10^{9} / \mathrm{L}\right)$ & $6.9 \pm 4.4$ & $5.6 \pm 3.3$ \\
Glutamic-pyruvic transaminase $(\mathrm{U} / \mathrm{L})$ & $43.1 \pm 6.3$ & $46.1 \pm 4.7$ \\
Serum creatinine $(\mu \mathrm{mol} / \mathrm{L})$ & $82.5 \pm 11.3$ & $93.5 \pm 10.4$ \\
Blood urea $(\mathrm{mmol} / \mathrm{L})$ & $6.9 \pm 1.7$ & $8.9 \pm 3.9$ \\
CEA (ng/mL) & $26.2 \pm 3.1$ & $13.3 \pm 5.2$ \\
Heart rate $($ beats $/ \mathrm{min})$ & $85.3 \pm 11.5$ & $80.9 \pm 15.1$ \\
Blood pressure $(\mathrm{mmHg})$ & $139.7 \pm 10.2$ & $137.5 \pm 11.3$ \\
\hline
\end{tabular}

IPTC, intrapleural perfusion thermo-chemotherapy. malfunctions were observed (Table 1).

Baseline CEA levels were high preoperatively in all patients, with a mean value of $26.2 \pm 3.1 \mathrm{ng} / \mathrm{mL}$. They markedly decreased 1 month after IPTC, showing a mean value of $13.3 \pm 5.2 \mathrm{ng} / \mathrm{mL}$.

Morphological characteristics of tumor cell apoptosis were obvious under light microscopy and TEM, including pyknosis and karyorrhexis (Figure 2).

Follow-up visits persisted until October 2014 for all patients. A total of 40 patients survived for more than 1 year, with 19, 7, and 4 surviving over 2, 3, and 4 years, respectively. Median survival time was 21.7 months; the one-year survival rate was $74.1 \%$.
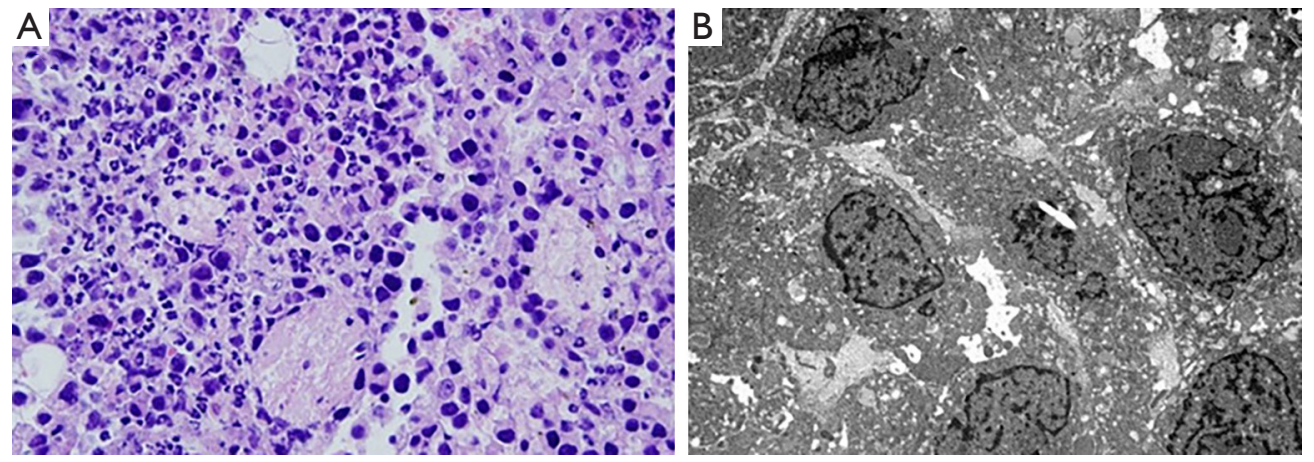

Figure 2 Changes in cell morphology. (A) Most human lung cancer cells exhibited pyknosis by light microscopy $\times 400$; (B) tumor cells showed karyorrhexis as evaluated by TEM, $\times 8,000$. 


\section{Discussion}

MPE, a common complication in patients with stage-IV lung cancer, can severely reduce the QOL and survival rate (11). Increased pleural fluid limits the mechanical dilation of the lungs, thereby affecting pulmonary and cardiac function; this usually results in severe shortness of breath and circulation failure. The International Association for the Study of Lung Cancer (IASLC) reported a 1-year survival rate of $36 \%$ in patients with carcinomatous pleuritis (12). Currently, the primary treatment for pleural fluid is local therapy (e.g., intrathoracic injection of chemotherapy drugs, solidifiers and bioactive agents). However, repeated operations often cause more pain to the patients and increase body exhaustion, and the reduction of thoracic effusion is not satisfactory. The traditional surgical therapy, including pleuropneumonectomy, often causes various complications and results in a high death rate; it is therefore not suitable for many patients (13).

It is a consensus in modern medicine that cancer cells would be killed when heated to $41.0-45.0{ }^{\circ} \mathrm{C}$ for dozens of minutes (14). Cancer cells are supported by abnormal capillaries and cannot store oxygen efficiently. The characteristics of cancer cells make them susceptible to heating (e.g., $42.0^{\circ} \mathrm{C}$ ), which considerably blocks or reduces their metabolism, as well as the activities of enzymes involved in cell division and DNA and RNA syntheses $(15,16)$. Hyperthermia constitutes a highly effective tool for cancer treatment, particularly in combination with chemotherapy, radiotherapy or immunotherapy, which show synergetic effects (17-19). We perfused the pleural cavity with heated saline containing cisplatin through mechanical circulation, which resulted in the death of residual suspension cancer cells and low metastasis on the thoracic membrane, thereby blocking or reducing the pleural fluid.

In intrapleural thermo-chemotherapy, the chemotherapeutic drugs to be injected into the pleural cavity are diluted to about $3,000 \mathrm{~mL}$ and warmed to $43{ }^{\circ} \mathrm{C}$. The drugs are quickly injected into the pleural cavity, and induce apoptosis of suspension cancer cells in the pleural cavity and low tumor metastasis. In this manner, the therapy can effectively reduce pleural effusion and increase survival.

In this study, we applied thoracoscopy under the VATS approach, which allows minimally invasive operation for lung decortication. Besides, the lung can be dilated after fiberboard removal; this distributes thermo-therapy more evenly within the pleural cavity, making it easier for the drugs to penetrate into the tissues, thereby enhancing the therapeutic effectiveness. Meanwhile, thoracoscopy can detect abnormalities in the lungs, pleural space and diaphragm, and can guide biopsy for pathological diagnosis and provide a pathological foundation for comprehensive treatment after surgery.

Overall, thermo-chemotherapy for lung cancer and MPE under video-assisted thoracoscope can help reach accurate pathological diagnosis and considerably reduce the effusion in a short period of time. Furthermore, this therapy is minimally invasive with few side effects, and can prolong patient survival. The above advantages of the current therapy strongly support broader application.

\section{Acknowledgements}

None.

\section{Footnote}

Conflicts of Interest: The authors have no conflicts of interest to declare.

Ethical Statement: This study was approved by the ethics committee of Hangzhou First People's Hospital (No. 101-01 Ethics).

\section{References}

1. Morgensztern D, Waqar S, Subramanian J, et al. Prognostic impact of malignant pleural effusion at presentation in patients with metastatic non-small-cell lung cancer. J Thorac Oncol 2012;7:1485-9.

2. Biaoxue R, Hui P, Wenlong G, et al. Evaluation of efficacy and safety for recombinant human adenovirus-p 53 in the control of the malignant pleural effusions via thoracic perfusion. Sci Rep 2016;6:39355.

3. Zarogoulidis $\mathrm{P}$, Chatzaki E, Hohenforst-Schmidt W, et al. Management of malignant pleural effusion by suicide gene therapy in advanced stage lung cancer: a case series and literature review. Cancer Gene Ther 2012;19:593-600.

4. Matsuzaki Y, Edagawa M, Shimizu T, et al. Intrapleural hyperthermic perfusion with chemotherapy increases apoptosis in malignant pleuritis. Ann Thorac Surg 2004;78:1769-72.

5. Hofmann HS, Wiebe K. Cytoreductive surgery and hyperthermic intrathoracic chemotherapy perfusion. Chirurg 2010;81:557-62.

6. Ishibashi H, Kobayashi M, Takasaki C, et al. Interim 
results of pleurectomy/decortication and intraoperative intrapleural hyperthermic cisplatin perfusion for patients with malignant pleural mesothelioma intolerable to extrapleural pneumonectomy. Gen Thorac Cardiovasc Surg 2015;63:395-400.

7. Migliore M, Calvo D, Criscione A, et al. Cytoreductive surgery and hyperthermic intrapleural chemotherapy for malignant pleural diseases: preliminary experience. Future Oncol 2015;11:47-52.

8. Ceppa DP, Kosinski AS, Berry MF, et al. Thoracoscopic lobectomy has increasing benefit in patients with poor pulmonary function: a Society of Thoracic Surgeons Database analysis. Ann Surg 2012;256:487-93.

9. Matsuzaki Y, Shibata K, Yoshioka M, et al. Intrapleural perfusion hyperthermo-chemotherapy for malignant pleural dissemination and effusion. Ann Thorac Surg 1995;59:127-31.

10. Kang M, Zhou L, Lin P. Treatment of pleural effusion caused by lung carcinoma with circular intrapleural hyperthermic perfusion and its mechanism. Zhonghua Yi Xue Za Zhi 2001;81:1176-9.

11. Yokoi K, Matsuguma H. Surgical treatment of lung cancer with carcinomatous pleuritis. Nihon Geka Gakkai Zasshi 2013;114:196-200.

12. Fukui T, Yokoi K. The role of surgical intervention in

Cite this article as: Hu R, Jiang H, Li H, Wei D, Wang G, Ma S. Intrapleural perfusion thermo-chemotherapy for pleural effusion caused by lung carcinoma under VATS. J Thorac Dis 2017;9(5):1317-1321. doi: 10.21037/jtd.2017.04.65 lung cancer with carcinomatous pleuritis. J Thorac Dis 2016;8:S901-7.

13. Go T, Misaki N, Matsuura N, et al. Role of surgery in multi-modality treatment for carcinomatous pleuritis in patients with non-small cell lung cancer. Surg Today 2015;45:197-202.

14. Kim HC, Kim E, Jeong SW, et al. Magnetic nanoparticleconjugated polymeric micelles for combined hyperthermia and chemotherapy. Nanoscale 2015;7:16470-80.

15. Hu R, Ma S, Li H, et al. Effect of magnetic fluid hyperthermia on lung cancer nodules in a murine model. Oncol Lett 2011;2:1161-4.

16. Speit G, Schütz P. Hyperthermia-induced genotoxic effects in human A549 cells. Mutat Res 2013;747-748:1-5.

17. Zhao P, Jiang H, Su D, et al. Inhibition of cell proliferation by mild hyperthermia at $43^{\circ} \mathrm{C}$ with Paris Saponin I in the lung adenocarcinoma cell line PC-9. Mol Med Rep 2015;11:327-32.

18. Wust P, Hildebrandt B, Sreenivasa G, et al. Hyperthermia in combined treatment of cancer. Lancet Oncol 2002;3:487-97.

19. Saga T, Sakahara H, Nakamoto Y, et al. Enhancement of the therapeutic outcome of radio-immunotherapy by combination with wholebody mild hyperthermia. Eur J Cancer 2001;37:1429-34. 